\title{
NOTE ON COUNTEREXAMPLES IN STRONG UNIQUE CONTINUATION PROBLEMS
}

\author{
THOMAS H. WOLFF
}

(Communicated by J. Marshall Ash)

\begin{abstract}
There are smooth functions on $\mathbb{R}^{d}$ vanishing to infinite order at a point and satisfying the differential inequality $|\Delta u| \leq V|u|$ with $V \in$ weak $L^{d / 2}$, and with $V \in L^{1}$ if $d=2$.
\end{abstract}

\section{INTRODUCTION}

A differential equation or inequality has the strong unique continuation property (SUCP) if every $C^{\infty}$ solution vanishing to infinite order at a point vanishes identically. Jerison and Kenig [5] proved that in $\mathbb{R}^{d}$ with $d \geq 3, V \in L_{\mathrm{loc}}^{d / 2}$ implies

$$
|\Delta u| \leq V|u|
$$

has the strong unique continuation property. The exponent $d / 2$ is sharp $(u=$ $\exp \left(-|x|^{-\varepsilon}\right)$ ), but the $L^{d / 2}$ condition can be replaced with certain other conditions having the same scale $[2,8]$. We're concerned here with a result of Stein [8] that (1) has the SUCP if $V$ is weak $L_{\text {loc }}^{d / 2}$ with small norm, i.e. if

$$
\sup _{a \in \mathbb{R}^{d}} \lim _{\varepsilon \rightarrow 0} \sup _{\lambda} \lambda^{d / 2} \mid\{x:|x-a|<\varepsilon \text { and } V(x)>\lambda\} \mid
$$

is sufficiently small. We will show that in this result of Stein, the "sufficiently small" condition is needed.

Theorem 1. For $d \geq 3$ there is $u: \mathbb{R}^{d} \rightarrow \mathbb{R}$, smooth and not identically zero, and vanishing to infinite order at the origin, such that $|\Delta u| \leq V|u|$ with $V \in$ weak $L^{d / 2}$, i.e.

$$
\sup _{\lambda>0} \lambda^{d / 2}\left|\left\{x \in \mathbb{R}^{d}: V(x)>\lambda\right\}\right|<\infty .
$$

It turns out that the $\mathbb{R}^{2}$ version of the same construction disproves the $\mathbb{R}^{2}$ analogue of the Jerison and Kenig result.

Received by the editors April 3, 1989.

1980 Mathematics Subject Classification (1985 Revision). Primary 35J05.

Research supported in part by NSF Grant DMS-87-03456. 
Theorem 2. There is $u: \mathbb{R}^{2} \rightarrow \mathbb{R}$ smooth and not identically zero, vanishing to infinite order at the origin and such that $|\Delta u| \leq V|u|$ with $V \in L^{1}$.

Remarks. (1) The examples have to be a bit more complicated than $e^{-|x|^{-\varepsilon}}$; e.g., it can be shown that $|\Delta u| \leq V|u|$ has the SUCP if $V$ is weak $L^{d / 2}$ and radial.

(2) There are some other open questions about the Laplacian and the SUCP, e.g., whether the SUCP holds for $|\Delta u| \leq V|u|$ with "Kato-class" potentials $V$ (see e.g. [2]) and whether it holds for $|\Delta u| \leq V|\nabla u|$ with $V \in L^{d}$ (see [1,6]). Our construction does not answer those questions. See the remark at the end of the paper.

\section{PROOF OF THE THEOREMS}

Let $\mathbf{e} \in \mathbb{R}^{d}$ be a fixed unit vector and let $\Gamma(x)=|x-\mathbf{e}|^{-(d-2)}$ if $d \geq 3$, $\Gamma(x)=\log \frac{1}{|x-\mathbf{e}|}$ if $d=2$. Let $p_{n-1}$ be the degree $n-1$ Taylor polynomial of $\Gamma$ at the origin and let $f_{n}=\Gamma-p_{n-1}$.

Note that the functions $f_{n}$ already "explain" the difference between small and large weak $L^{d / 2}$ norms. Let $\bar{f}_{n}$ be obtained from $f_{n}$ by smoothing off on a disc $D$ of radius $\varepsilon \ll \frac{1}{n}$ centered at e. Then $\bar{f}_{n}$ has the following properties:

(i) $\bar{f}_{n}=\mathscr{O}\left(|x|^{n-1}\right)$ at $\infty$;

(ii) $\bar{f}_{n}=\mathscr{O}\left(|x|^{n}\right)$ at 0 ;

(iii) $\Delta \bar{f}_{n} \in C_{0}^{\infty}\left(\mathbb{R}^{d} /\{0\}\right)$.

Moreover, $\Delta \bar{f}_{n} / \bar{f}_{n}$ is essentially $\varepsilon^{-2}$ on $D$ and zero otherwise, and therefore belongs to $L^{d / 2}$ with norm independent of $n$; on the other hand, the Carleman inequality of Stein [8] implies a lower bound on the weak $L^{d / 2}$ norm of any function satisfying (i)-(iii).

Going back to the proof, we have the following "gluing lemma."

Lemma. Suppose $n \in \mathbb{Z}^{+}, \varepsilon>0$. If $r$ is large enough and $s>0$ is small enough, then there is a smooth function $g=g_{n \varepsilon}^{r s}: \mathbb{R}^{d} /\{$ re, se $\} \rightarrow \mathbb{R}$ such that

$$
\begin{array}{ll}
g(x)=r^{n} f_{n}\left(r^{-1} x\right) & \text { when }|x|>2, \\
g(x)=-s^{n} f_{n+1}\left(s^{-1} x\right) & \text { when }|x|<\frac{1}{2}, \\
|\Delta g|<\varepsilon|g| & \text { when } \frac{1}{2} \leq|x| \leq 2 .
\end{array}
$$

Moreover $g$ satisfies the following estimates when $|\alpha|<n,\left(1-\frac{1}{n+2}\right) s \leq|x| \leq$ $\left(1-\frac{1}{n+1}\right) r$ :

$$
\left|D^{\alpha} g\right| \leq \begin{cases}C_{n \alpha}|x|^{n-|\alpha|}, & \text { if }\left|\frac{x}{s}-\mathbf{e}\right|>\frac{1}{n+2} \\ C_{\alpha} s^{n-|\alpha|}\left|\frac{x}{s}-\mathbf{e}\right|^{-(d-2+|\alpha|)}, & \text { if }\left|\frac{x}{s}-\mathbf{e}\right|<\frac{1}{n+2} .\end{cases}
$$

Here we replace $\left|\frac{x}{s}-\mathbf{e}\right|^{-(d-2+|\alpha|)}$ by $\log \left(1 /\left|\frac{x}{s}-\mathbf{e}\right|\right)$ if $d-2=|\alpha|=0$.

Proof. Let $Z_{k}$ be the term of homogeneity $k$ in the expansion of $\Gamma$ at zero. A standard estimate (e.g. [9], p. 167, Equation 7.33.6) is then that $\left|Z_{k}(x)\right| \leq$ $C k^{d-3}|x|^{k}$, and considering $Z_{k}$ as a harmonic function on a ball of radius $\frac{|x|}{k}$, it follows that $\left|D^{\alpha} Z_{k}(x)\right| \leq C_{\alpha} k^{d-3+|\alpha|}|x|^{k-|\alpha|}$. The expansion of $r^{n} f_{n}\left(r^{-1} x\right)$ at zero is

$$
\sum_{k \geq n} r^{n-k} Z_{k}(x)
$$


Choosing $r$ large, we can make $r^{n} f_{n}\left(r^{-1} x\right)-Z_{n}(x)$ and its first four derivatives arbitrarily small on $\frac{1}{4}<|x|<4$. Also

$$
-s^{n} f_{n+1}\left(s^{-1} x\right)=-s^{n}\left|s^{-1} x-\mathbf{e}\right|^{-(d-2)}+\sum_{k=0}^{n} s^{n-k} Z_{k}(x) .
$$

If $s$ is small, $-s^{n} f_{n+1}\left(s^{-1} x\right)-Z_{n}(x)$, and its first four derivatives will be small when $\frac{1}{4}<|x|<4$. Now let $\Psi$ be $C^{\infty}$, with $\Psi=1$ when $|x|<\frac{1}{2}, \Psi=0$ when $|x|>2$, and

$$
\tilde{g}(x)=r^{n} f_{n}\left(r^{-1} x\right)+\Psi(x)\left(-s^{n} f_{n+1}\left(s^{-1} x\right)-r^{n} f_{n}\left(r^{-1} x\right)\right) .
$$

Then $\tilde{g}$ satisfies the first two requirements of the lemma and is $C^{4}$ close to $Z_{n}$ on $\frac{1}{4}<|x|<4$. Since $Z_{n}\left(\frac{x}{|x|}\right)$ is a solution of a second order ODE, we know that $\nabla Z_{n}$ vanishes only at zero. So we have a lower bound on $|\nabla \tilde{g}|$ for $\frac{1}{4}<|x|<4$, provided that $r, s^{-1}$ are large. Let $M$ be the zero set of $\tilde{g}$ and $\delta(x)=\operatorname{dist}(x, M)$. For suitable $\rho_{0}, \delta$ is smooth on $\left\{x: \delta(x)<\rho_{0}\right\}$ (tubular neighborhood theorem) with bounds on its derivatives, and this is uniform in $r, s$ as $r, s^{-1} \rightarrow \infty$. Let $\chi: R \rightarrow \mathbb{R}$ be smooth with $\chi(t)=1$ when $|t|<\frac{1}{2} \rho_{0}$, $\chi(t)=0$ when $|t|<\rho_{0}$, and let

$$
g=\tilde{g}-\frac{1}{2} \delta^{2} \chi \circ \delta \Delta \tilde{g} .
$$

If we differentiate $g$ by the product rule, we obtain

$$
\Delta g=\left(\Delta \tilde{g}-\Delta\left(\frac{1}{2} \delta^{2}\right) \chi \circ \delta \Delta \tilde{g}\right)-2 \nabla\left(\frac{1}{2} \delta^{2}\right) \cdot \nabla(\chi \circ \delta \Delta \tilde{g})-\frac{1}{2} \delta^{2} \Delta(\chi \circ \delta \Delta \tilde{g})
$$

Since $\Delta\left(\frac{1}{2} \delta^{2}\right)=1$ on $M$, it is clear that all three terms are

$$
\leq C \delta\|\Delta \tilde{g}\|_{C^{2}},
$$

with $C$ independent of $r$ and $s$. On the other hand, since $|\nabla \tilde{g}|$ is bounded away from zero and $|\nabla \nabla \tilde{g}|$ is bounded, we know that $|\tilde{g}| \geq C^{-1} \delta$ when $\frac{1}{4}<$ $|x|<4$. So

$$
|g|>C^{-1} \delta-\frac{1}{2}\|\Delta \tilde{g}\|_{\infty} \chi \circ \delta \delta^{2} .
$$

Since $Z_{n}$ is harmonic, we make $\|\Delta \tilde{g}\|_{C^{2}}$ arbitrarily small by taking $r, s^{-1}$ large. Then (5) implies $|g|>\frac{1}{2} C^{-1} \delta$ when $\frac{1}{4}<|x|<4$, so (4) becomes

$$
|\Delta g| \leq C\|\Delta \tilde{g}\|_{C^{2}}|g| \leq \varepsilon|g| \text {. }
$$

When $\frac{1}{2}<|x|<2$, the last statement of the lemma follows from the construction-all that is being claimed is that the derivative bounds are independent of $r$ and $s$ as $r, s^{-1} \rightarrow \infty$. When $|x|>2$ or $|x|<\frac{1}{2}$, the last statement follows from (2) or (3) respectively, using $\left|D^{\alpha} Z_{k}\right| \leq C_{\alpha} k^{d-3+|\alpha|}|x|^{k-|\alpha|}$.

We now smooth off the singularity of $g_{n \varepsilon}^{r s}$ at $s \mathbf{e}$. The estimate $\left|Z_{k}\right|<$ $C k^{d-3}|x|^{k}$ implies that $\left|f_{n}(x)\right| \geq \frac{1}{2}|x-\mathbf{e}|^{-(d-2)}\left(\frac{1}{2} \log \frac{1}{|x-\mathbf{e}|}\right.$ if $\left.d=2\right)$, provided that $(n+1)|x-\mathbf{e}|$ is less than a suitable constant $b<1$. Moreover, for $\rho<\frac{b}{n+1}$ the variation of $f_{n}$ on the set $\left\{x: \frac{1}{2} \rho<|x-\mathbf{e}|<\rho\right\}$ is $\leq C \rho^{2-d}$ for all $d$ (i.e. $\leq C$ when $d=2$; this follows since $\left|\nabla Z_{k}\right| \leq C|x|^{k}$ ). Let $\Psi$ be 
smooth with $0 \leq \alpha \leq 1$ and $\psi(x)=1$ when $|x-\mathbf{e}|<\frac{1}{2}, \Psi(x)=0$ when $|x-\mathbf{e}|>1$. For $\rho<\frac{b}{n+1}$ choose a point $x_{0}$ with $\left|\frac{x_{0}}{s}-\mathbf{e}\right|=\rho$ and define

$$
g_{n \varepsilon \rho}^{r s}(x)=\dot{g}_{r \varepsilon}^{r s}(x)+\Psi\left(\rho^{-1}\left(\frac{x}{s}-\mathbf{e}\right)\right)\left(g_{n \varepsilon}^{r s}\left(x_{0}\right)-g_{n \varepsilon}^{r s}(x)\right) ;
$$

if $d=2$, use $\log \frac{1}{\rho}$ instead of $\rho^{-(d-2)}$. Then $C^{-1} s^{n} \rho^{-(d-2)} \leq\left|g_{n \varepsilon \rho}^{r s}\right| \leq$ $C s^{n} \rho^{-(d-2)}$ for $\left|\frac{x}{s}-\mathbf{e}\right|<\rho$ if $d \geq 3$, and $C^{-1} s^{n} \log \frac{1}{\rho} \leq\left|g_{n \varepsilon \rho}^{r s}\right| \leq C s^{n} \log \frac{1}{\rho}$ for $\left|\frac{x}{s}-\mathbf{e}\right|<\rho$ if $d=2$. Using the lemma, the remark about the variation of $f_{n+1}$ and the product rule, if $|\alpha| \geq 1$ and $\left|\frac{x}{s}-\mathbf{e}\right|<\rho$, then

$$
\left|D^{\alpha} g_{n \varepsilon \rho}^{r s}\right| \leq C_{\alpha} s^{n-|\alpha|} \rho^{-(d-2+|\alpha|)} .
$$

This implies in particular that for $\left|\frac{x}{s}-\mathbf{e}\right|<\rho$,

$$
\begin{gathered}
\left|\Delta g_{n \varepsilon \rho}^{r s}\right| \leq C(\rho s)^{-2}\left|g_{n \varepsilon \rho}^{r s}\right| \quad \text { if } d \geq 3, \\
\left|\Delta g_{n \varepsilon \rho}^{r s}\right| \leq C(\rho s)^{-2}\left(\log \frac{1}{\rho}\right)^{-1}\left|g_{n \varepsilon \rho}^{r s}\right| \quad \text { if } d=2 .
\end{gathered}
$$

Moreover, the previous bound and the lemma give

$$
\left|D^{\alpha} g_{n \varepsilon \rho}^{r s}\right| \leq A_{n \alpha \rho}|x|^{n-|\alpha|}
$$

for $|\alpha| \geq 0,\left(1-\frac{1}{n+2}\right) s \leq|x| \leq\left(1-\frac{1}{n+1}\right) r$.

Now suppose $\left\{\rho_{n}\right\}_{1}^{\infty}$ is any sequence with $\rho_{j}<\frac{b}{j+1}$. Let $r_{1}=1$ and define $u(x)$ for $|x| \geq \frac{1}{2}$ by $u(x)=-f_{1}(x)$, smoothed off slightly when $|x-\mathbf{e}|<\rho_{1}$, so as to be $C^{\infty}$. For $j \geq 2$ we will define recursively numbers $\eta_{j}$ and $r_{j}$ with $r_{j}<\eta_{j}<r_{j-1}$ and the values of the function $u$ on the set $E_{j}=\left\{x:\left(1-\frac{1}{j+1}\right) r_{j} \leq\right.$ $\left.|x| \leq\left(1-\frac{1}{j}\right) r_{j-1}\right\}$. Namely, if this has been done for $j \leq n$, then make $\eta_{n+1} / r_{n}$ and $r_{n+1} / \eta_{n+1}$ small enough that a function $g_{n 2^{-n}}^{r_{n} / \eta_{n+1} r_{n+1} / \eta_{n+1}}$ as in the lemma exists, and so that (with $A_{n \alpha \rho}$ as in (8))

$$
A_{n+1 \alpha \rho_{n+2}}\left(r_{1} \cdots r_{n}\right)^{-1} r_{n+1}^{n-|\alpha|}<2^{-n}
$$

for $|\alpha|<\frac{n}{2}$. Then define $u$ on $E_{n+1}$ by

$$
u(x)=C_{n+1} g_{n 2-n}^{r_{n} / \eta_{n+1} r_{n+1} / \eta_{n+1}}\left(\eta_{n+1}^{-1} x\right),
$$

where the constant $C_{n+1}$ is chosen to make the definition consistent when $|x|=$ $\left(1-\frac{1}{n+1}\right) r_{n}$. This works out to $C_{2}=-\eta_{2}$ and $C_{n+1}\left(\frac{r_{n}}{\eta_{n+1}}\right)^{n}=-C_{n}\left(\frac{r_{n}}{\eta_{n}}\right)^{n-1}$; i.e., $C_{n+1}=(-1)^{n}\left(r_{1} \cdots r_{n}\right)^{-1} \eta_{n+1}^{n}$. There are two conditions to check.

(i) $u$ vanishes to infinite order at zero.

Substituting the value of $C_{n}$ into (9) and using (8), on $E_{n+1}$

$$
\left|D^{\alpha} u\right| \leq A_{n \alpha \rho_{n+1}}\left(r_{1} \cdots r_{n}\right)^{-1}|x|^{n-\alpha} \leq A_{n \alpha \rho_{n+1}}\left(r_{1} \cdots r_{n-1}\right)^{-1} r_{n}^{n-|\alpha|-1}<2^{-(n-1)}
$$

for $|\alpha|<\frac{n}{2}$, by choice of $r_{n}$. Statement (i) follows.

(ii) If $\left\{\rho_{n}\right\}$ are chosen appropriately, then $\frac{\Delta u}{u} \in$ weak $L^{d / 2}$ if $d \geq 3$ and $\frac{\Delta u}{u} \in L^{1}$ if $d=2$.

$\Delta u$ vanishes except on $\bigcup_{n}\left(B_{n} \cup D_{n}\right)$ where $B_{n}=\left\{x: \frac{1}{2} \eta_{n} \leq|x| \leq 2 \eta_{n}\right\}$ 
and $D_{n}=\left\{x:\left|\frac{x}{r_{n}}-\mathbf{e}\right|<\rho_{n}\right\}$. We have $\left|\Delta g_{n 2^{-n}}^{r s}\right| \leq 2^{-n}\left|g_{n 2^{-n}}^{r s}\right|$, and therefore $|\Delta u| \leq C \eta_{n}^{-2} 2^{-n}|u|$ on $B_{n}$.

So

$$
\int_{\cup B_{n}}\left(\frac{\Delta u}{u}\right)^{d / 2} \leq C \sum 2^{-n \times d / 2}<\infty .
$$

If $d \geq 3$ we simply take $\rho_{n}=\frac{b}{n+1}$. Let $E(\lambda)$ be the distribution function of $\frac{\Delta u}{n}\left\lceil\bigcup_{n} D_{n} . D_{n}\right.$ is a disc of radius $\frac{b}{n+1} r_{n}$ and $|\Delta u| \leq C\left(\frac{b}{n+1} r_{n}\right)^{-2}|u|$ there by (6). So

$$
E(\lambda) \leq C \sum_{C\left(\frac{b}{n+1} r_{n}\right)^{-2}>\lambda}\left(\frac{b}{n+1} r_{n}\right)^{d} \leq C \lambda^{-d / 2},
$$

since the $r_{n}$ are decreasing at least geometrically.

If $d=2$ we choose $\rho_{n}$ so that $\sum\left(\log \frac{1}{\rho_{n}}\right)^{-1}<\infty$. Then by (7),

$$
\int_{B_{n}}\left|\frac{\Delta u}{u}\right| \leq C \sum\left(\rho_{n} r_{n}\right)^{2}\left(\rho_{n} r_{n}\right)^{-2} \log \frac{1}{\rho_{n}}<\infty .
$$

Remark. The reason it was possible to do such a simple construction is that the Carleman inequalities, which would prove the opposite of Theorems 1 and 2, fail for a very simple reason: the relevant Sobolev inequalities are already false. For example, in $\mathbb{R}^{2}$, convolution with $\Gamma$ does not map $L^{1}$ to $L^{\infty}$. This fact allowed us to do our construction very close to the diagonal (where $\left.\left|x-r_{n} \mathbf{e}\right|<\frac{b}{n+1} r_{n}\right)$ and avoid considering cancellations. In the problems mentioned in Remark (2) of the Introduction, the Carleman inequalities fail for a different reason, discovered by Jerison [4] (see also [1]), and concerning what happens in the region $|x-y| \simeq \frac{|y|}{\sqrt{n}}$ where the relevant kernels have oscillatory behavior. See e.g. [7] and [10] for various constructions of kernels.

\section{ACKNOWLEDGMENT}

I thank M. Christ for a conversation which clarified a technical point in the proof.

\section{REFERENCES}

1. B. Barcelo, C. E. Kenig, A. Ruiz, and D. C. Sogge, Weighted Sobolev inequalities and unique continuation for the Laplacian plus lower order terms, Illinois J. Math. 32 (1988), 230-245.

2. S. Chanillo and E. Sawyer, Unique continuation for $\Delta+V$ and the C. Fefferman-Phong class, Trans. Amer. Math. Soc. 318 (1990), 275-300.

3. E. Fabes, N. Garofalo, and F. H. Lin, (to appear).

4. D. Jerison, Carleman inequalities for the Dirac and Laplace operators and unique continuation, Adv. in Math. 62 (1986), 118-134.

5. D. Jerison and C. Kenig, Unique continuation and absence of positive eigenvalues for Schröding-operators, Ann. of Math. (2) 12 (1985), 463-494.

6. Y.-M. Kim, Ph.D. Thesis, Massachusetts Institute of Technology, 1989.

7. C. Sogge, A strong unique continuation theorem for second order elliptic differential equations, Amer. J. Math. 112 (1990), 943-984.

8. E. Stein, Appendix to "Unique continuation," Ann. of Math. (2) 121 (1985), 489-494. 
9. G. Szego, Orthogonal polynomials, Amer. Math. Soc. Colloq. Publ., Vol. 23, Amer. Math. Soc., Providence, RI, 1939.

10. T. Wolff, Unique continuation for $|\Delta u| \leq V|\nabla u|$ and related problems, Revista Math. Iberoamericana 6 (1990), 155-200.

Department of Mathematics, California Institute of Technology, Pasadena, CaliFORNIA 91125 